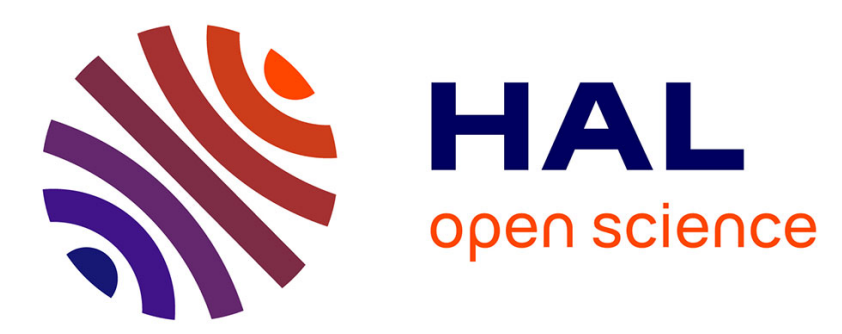

\title{
MARSIS surface reflectivity of the south residual cap of Mars
}

Jeremie Mouginot, W. Kofman, A. Safaeinili, C. Grima, A. Null Herique, J.J. Plaut

\section{- To cite this version:}

Jeremie Mouginot, W. Kofman, A. Safaeinili, C. Grima, A. Null Herique, et al.. MARSIS surface reflectivity of the south residual cap of Mars. Icarus, 2009, 201 (2), pp.454. 10.1016/j.icarus.2009.01.009 . hal-00533497

\section{HAL Id: hal-00533497 https://hal.science/hal-00533497}

Submitted on 7 Nov 2010

HAL is a multi-disciplinary open access archive for the deposit and dissemination of scientific research documents, whether they are published or not. The documents may come from teaching and research institutions in France or abroad, or from public or private research centers.
L'archive ouverte pluridisciplinaire HAL, est destinée au dépôt et à la diffusion de documents scientifiques de niveau recherche, publiés ou non, émanant des établissements d'enseignement et de recherche français ou étrangers, des laboratoires publics ou privés. 


\section{Accepted Manuscript}

MARSIS surface reflectivity of the south residual cap of Mars

Jeremie Mouginot, W. Kofman, A. Safaeinili, C. Grima, A. Herique, J.J. Plaut

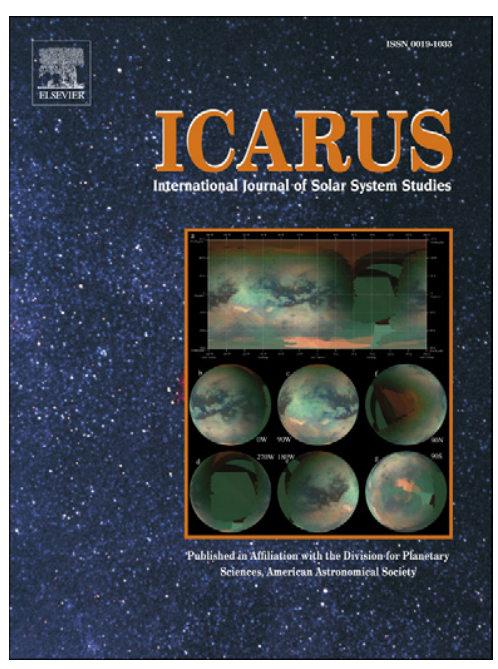

PII: $\quad$ S0019-1035(09)00031-1

DOI: $\quad$ 10.1016/j.icarus.2009.01.009

Reference: $\quad$ YICAR 8891

To appear in: Icarus

Received date: 1 July 2008

Revised date: 16 October 2008

Accepted date: 9 January 2009

Please cite this article as: J. Mouginot, W. Kofman, A. Safaeinili, C. Grima, A. Herique, J.J. Plaut, MARSIS surface reflectivity of the south residual cap of Mars, Icarus (2009), doi:

10.1016/j.icarus.2009.01.009

This is a PDF file of an unedited manuscript that has been accepted for publication. As a service to our customers we are providing this early version of the manuscript. The manuscript will undergo copyediting, typesetting, and review of the resulting proof before it is published in its final form. Please note that during the production process errors may be discovered which could affect the content, and all legal disclaimers that apply to the journal pertain. 


\section{MARSIS surface reflectivity of the south residual cap}

\section{2 of Mars}

3 Mouginot J. ${ }^{1}$, (jeremie.mouginot@obs.ujf-grenoble.fr)

$4 \quad$ Kofman W. ${ }^{1}$, (wlodek.kofman@obs.ujf-grenoble.fr)

$5 \quad$ Safaeinili A. ${ }^{2}$, (ali.safaeinili@jpl.nasa.gov)

$6 \quad$ Grima C. ${ }^{1}$, (cyril.grima@obs.ujf-grenoble.fr)

$7 \quad$ Herique A. ${ }^{1}$, (alain.herique@obs.ujf-grenoble.fr)

$8 \quad$ Plaut J. J.2 (plaut@jpl.nasa.gov)

9

10

$11{ }^{1}$ Laboratoire de Planétologie de Grenoble, CNRS/UJF 38041 Grenoble Cedex, France

$12{ }^{2}$ Jet Propulsion Laboratory, California Institute of Technology, Pasadena, CA 91109.

13

14

15

16

17

18

19

20

21

22

23

Manuscript : 13 pages

Figures : 5 pages

Tables : 1 page 
24 Proposed running head:

25 MARSIS surface reflectivity of the South Residual Cap.

26

27 The name and address to which editorial correspondence and proofs should be 28 directed:

29 Jeremie MOUGINOT

$30 \quad 122$ rue de la piscine

3138000 Grenoble

32 FRANCE

$33 \quad$ Jeremie.mouginot@obs.ujf-grenoble.fr

34 
35

36

\section{(Abstract)}

The south residual cap of Mars is commonly described as a thin and bright layer of $\mathrm{CO}_{2}$-ice. The Mars Advanced Radar for Subsurface and Ionospheric Sounding (MARSIS) is a low-frequency radar on board Mars Express operating at the wavelength between 55 and $230 \mathrm{~m}$ in vacuum. The reflection of the radar wave on a stratified medium like the residual cap can generate interferences, causing weaker surface reflections compared to reflections from a pure water ice surface.

In order to understand this anomalous low reflectivity, we propose a stratified medium model, which allows us to estimate both the thickness and the dielectric constant of the optically thin slab. First, we consider the residual cap as single unit and show that the decrease in the reflected echo strength is well explained by a mean thickness of $11 \mathrm{~m}$ and a mean dielectric constant of 2.2. This value of dielectric constant is close to the experimental value 2.12 for pure $\mathrm{CO}_{2}$-ice. Second, we study the spatial variability of the radar surface reflectivity. We observe that the reflectivity is not homogeneous over the residual cap. This heterogeneity can be modeled either by variable thickness or variable dielectric constant. The surface reflectivity shows that two different units comprise the residual cap, one central unit with high reflectivity and surrounding, less reflective units.

KEYWORD: RADAR OBSERVATIONS, MARS, POLAR CAPS, ICES 


\section{Introduction}

56

57

58

59

60

61

62

63

64

65

66

67

68

69

70

71

72

73

74

75

76

\subsection{South residual cap}

The south polar plateau of Mars is partially covered by a perennial thin layer of carbon dioxide $\left(\mathrm{CO}_{2}\right)$ ice, which is easily visible from Earth and spacecraft due to its high albedo compared to the surrounding regions. Astronomers have observed this layer over a century [Flammarion 1892], but the composition $\left(\mathrm{CO}_{2}\right.$-ice $)$ was determined using Viking orbiter thermal data [Kieffer, 1979; Paige et al. 1990]. It has been shown that this $\mathrm{CO}_{2}$ layer directly lies above water ice $\left(\mathrm{H}_{2} \mathrm{O}\right.$-ice), which comprises the major part of the plateau [Plaut et al. 2007] in the form of South Polar Layered Deposits (SPLD).

Thermal data from Thermal Emission Imaging System (THEMIS) revealed the presence of small exposures of $\mathrm{H}_{2} \mathrm{O}$-ice adjacent to the $\mathrm{CO}_{2}$-ice, based on temperature signatures [Titus et al. 2003]. The Observatoire pour la Minéralogie, l'Eau, les Glaces, et l'Activité (OMEGA) observed spectral signatures of both $\mathrm{CO}_{2}$-ice and trace amounts of $\mathrm{H}_{2} \mathrm{O}$-ice within the residual ice cap [Bibring et al. 2004, Douté et al. 2007]. OMEGA also confirmed the identification of $\mathrm{H}_{2} \mathrm{O}$-ice-rich surfaces near the $\mathrm{CO}_{2}$-ice cap

4 The perennial $\mathrm{CO}_{2}$ deposit consists of numerous layers. Using Mars Global Surveyor 5 Mars Orbiter Camera (MOC) images, Thomas et al. [2005] showed that the south residual cap consists of two distinct layered units, which were deposited at different 
times, separated by a period of degradation. The older unit, about $10 \mathrm{~m}$ thick, has layers approximately $2 \mathrm{~m}$ thick. The younger unit has variable numbers of layers, each about $1 \mathrm{~m}$ thick.

An estimate of the quantity of $\mathrm{CO}_{2}$ in the slab, which can be compared to the total $\mathrm{CO}_{2}$ content of the atmosphere, was made by Byrne and Ingersoll [2003]. They showed that a $\mathrm{CO}_{2}$ residual ice cap with $10 \mathrm{~m}$ thickness, an area of $87,000 \mathrm{~km}^{2}$, and a density of $1.6 \mathrm{~g} . \mathrm{cm}^{-3}$, constitutes only about $5 \%$ of the average atmospheric mass.

\subsection{Surface Reflectivity measured by MARSIS}

MARSIS is a decameter-wave sounding radar, which can penetrate kilometers below the icy surface. It has provided important results on the Martian subsurface [Picardi et al. 2005; Plaut et al. 2007; Watters et al. 2007] and ionosphere [Gurnett et al. 2005 ;

Duru et al. 2006; Safaeinili et al. 2007; Espley et al. 2007]. The radar uses four frequency bands, which are centered at 1.8, 3, 4 and $5 \mathrm{MHz}(166,100,75$, and $60 \mathrm{~m}$ wavelength). Each band has a width of $1 \mathrm{MHz}$.

The data have been corrected for the distortion (phase shift) [Safaeinili et al. 2003; Mouginot et al. 2008a] and absorption [Mouginot et al. 2008b] due to the ionosphere. The radar frequency is close to the plasma frequency (up to4 MHz) of the ionosphere [Nagy et al. 2004; Gurnett et al. 2005] and as a result the signal is broadened significantly in addition to being delayed. This broadening of the pulses can cause smearing of the resulting radargram. Correction for ionospheric effects is performed to re-sharpen the pulses and compensate for the absorption effects as described in Mouginot et al. [2008a]. 
103 We quantify the echo returned by the surface from MARSIS radargrams by localizing

104 the position in the radargram corresponding to the surface echo and measuring the

105 amplitude. The position corresponds to the surface elevation given by Mars Orbiter

106 Laser Altimeter (MOLA).

107 This surface echo amplitude (i.e., surface reflectivity) allows us to build reflectivity

108 maps in each MARSIS frequency band (map at $4 \mathrm{MHz}$ in Fig. 1a; maps at other

109 frequencies show the same type of features). The map resolution is $14.7 \mathrm{~km}$ per pixel

110 (about the MARSIS footprint width). For bands centered at 3, 4 and $5 \mathrm{MHz}$, we used

111305,464 and 539 orbits, respectively, to construct reflectivity maps. For crossing

112 tracks, we average the data from multiple measurements from MARSIS is a nadir-

113 looking radar and the Mars Express polar orbit does not allow us to sound the surface

114 poleward of about $87^{\circ} \mathrm{N}$ and $87^{\circ} \mathrm{S}$; this lack of data results in a gap centered at the

115 pole.

116 To first order, the reflectivity is inversely correlated with the surface roughness,

117 because the power reflected by a surface at nadir decreases with its roughness. Thus

118 the topographic variations at lateral scales comparable to and/or larger than the

119 MARSIS wavelength are affecting the signal. This is normal behavior due to the loss

120 of coherency of the radar signal.

121 A simulator of returned radar echoes from Mars was developed by Nouvel et al.

122 [2004]. This computationally efficient radar signal simulation is based on the use of

123 the Facet Method as surface modeling scheme. The slope and the large-scale

124 roughness effects are simulated using MOLA topography to predict the surface echo

125 amplitude in each point [Nouvel et al. 2004]. In this simulation, the reflectivity 
126

127

128

129

130

131

132

133

134

135

136

137

138

139

140

141

142

143

144 We built a first order model to describe reflectivity in the south residual cap. This 145

variability is only due to surface slopes, with an assumption of a single fixed surface dielectric constant. It allows distinguishing dielectric and topographic effects.

For each MARSIS radargram, we generate the corresponding radar simulation and extract the surface amplitude from simulated radargrams to obtain a simulated reflectivity map (Fig. 1b). We use identical procedures to generate both the simulated map and the data map (Fig. 1a).

With such a simulated map, we can correct for any reflectivity variations that are due to topographic effects. Indeed, the reflection coefficient (backscattering coefficient) $R$ can be written as the product of a dielectric constant function and a roughness function [Ulaby et al. 1986], which is independent of the dielectric constant. By normalizing the reflectivity map by the simulated one, we obtain a map proportional to the dielectric constant (Fig. 1c). This normalization consists of the difference of the power logarithms between data and simulated map. This normalized map reveals variations of surface reflectivity across our area of interest. Indeed, one can see that the region of the residual cap has very low reflectivity values (black box in Fig. 1c) compared to the other parts of the SPLD.

$$
\text { model allows us to compute the radar wave propagation in a stratified medium and }
$$
then obtain a corresponding reflectivity.

Previous work indicates that the south residual cap consists of a thin perennial slab of $\mathrm{CO}_{2}$-ice overlapping $\mathrm{H}_{2} \mathrm{O}$-ice. So we model the south residual cap reflectivity using three layers: the atmosphere, the $\mathrm{CO}_{2}$-ice and the $\mathrm{H}_{2} \mathrm{O}$-ice (Fig. 2). 
150 In a two-layer medium with refractive indices $n_{\mathrm{i}}$ and $n_{\mathrm{j}}$, the reflection coefficient for

151 normal incidence is given by the equation:

$$
r_{i j}=\frac{n_{j}-n_{i}}{n_{j}+n_{i}}(1)
$$

153 In a stratified medium (i.e., with three layers in our case), the reflection coefficient

154 equation may be conveniently expressed in terms of the corresponding coefficients $r_{12}$ 155 and $r_{23}$ associated with the reflection coefficients at the first and the second interface, 156 respectively [Born and Wolf 1959]:

$$
r=\frac{r_{12}+r_{23} e^{2 i \beta}}{1+r_{12} r_{23} e^{2 i \beta}}
$$

158 where $\beta=\frac{2 \pi}{\lambda} n_{2} h, h$ is the thickness of the intermediate layer and $\lambda$ is the wavelength of the incident wave. $r_{12}, r_{23}$ may be obtained by substituting equation 1 with the corresponding subscripts. This notation implies that $n_{1}, n_{2}$ and $n_{3}$ are respectively, the refractive index for the upper (atmosphere), intermediate $\left(\mathrm{CO}_{2}\right.$ slab) and lower $\left(\mathrm{H}_{2} \mathrm{O}\right.$-ice) layers (see Fig. 2). This model does not include any losses in the media, which, we believe, is a good approximation because for $\mathrm{CO}_{2}-$ and $\mathrm{H}_{2} \mathrm{O}$-ice losses are known to be weak. In addition polar MARSIS measurements typically show low losses [Plaut et al., 2007]. The refractive index $n_{i}$ corresponds to the square root of the real part of dielectric constant: $n_{i}=\sqrt{\varepsilon_{i}}$. monochromatic, we cannot limit ourselves to equation 2 to obtain the reflectivity. Instead, we have to calculate the reflectivity as:

$$
R=\max \left(\| \operatorname{IFFT}\left(S(f) r(f) S^{*}(f) \|^{2}\right)(3)\right.
$$


172 where $f$ is the frequency, $r$ is the reflection coefficient defined in equation 2 and $S$ is

173 the linearly modulated chirp signal of MARSIS. Equation 3 describes our method to

174 model the amplitude of the surface echo: we apply to an ideal transmitted signal

175 (chirp) the reflection coefficient $r(f)$. The Inverse Fast Fourier Transform (IFFT) gives

176 a time dependent signal that corresponds to the output of the matched filter of the

177 receiver.

178 In our model, we describe the $\mathrm{H}_{2} \mathrm{O}$-ice as compact pure water ice, which corresponds

179 to a dielectric constant $\varepsilon_{3}$ equal to 3.15 . This value of pure water ice for the dielectric

180 constant is probably a good assumption, as previous workers have shown that the

181 deposits of the south polar-layered deposits are composed of relatively clean water ice

182 [Plaut et al. 2007; Zuber et al. 2007]. Moreover laboratory experiments have shown

183 that the real component varies between 3.14 and 3.19 [Ulaby et al. 1986] for various

184 types of "dirty ices". In case of porous ice, the dielectric constant decreases. For 185 example, if the porosity of ice were equal to $10 \%$, then, using Maxwell Garnett

186 mixing formulas [Sihvola, 1999], the dielectric constant would be 2.87. The dielectric

187 constant of the atmosphere is set to 1.

188 Fig. 3 presents the model of reflectivity $R$ as function of the $\mathrm{CO}_{2}$ thickness, $h$, for

189 different values of $\mathrm{CO}_{2}$ dielectric constant. The two free parameters in our model are

$190 h$ and the dielectric constant of the central layer $\varepsilon_{2}$ (the $\mathrm{CO}_{2}$-ice). Both have an

191 impact on the inferred reflectivity. The $\mathrm{CO}_{2}$ thickness in the plot is limited to the $0-20$

192 m range because previous studies have shown that the global thickness is around 10 $193 \mathrm{~m}$.

194 First, we see on Fig. 3 that the reflectivity is minimal for a layer whose optical

195 thickness $n_{2} h$ is close to $\lambda_{0} / 4$ ( $\lambda_{0}$ is the central wavelength) and the reflectivity is 196 maximal when optical thickness is close to $\lambda_{0} / 2$ (Born and Wolf 1979). 
197 Second, the reflectivity is minimum or strictly equal to zero when the dielectric

198 constant of the intermediate layer is equal to $\sqrt{n_{1} n_{3}}$. In our case, this corresponds with

199 a dielectric constant of $\varepsilon_{2} \cong 1.77$.

\section{Data Analysis}

\subsection{Comparison to $\mathrm{H}_{2} \mathrm{O}$-ice}

202 As the reflectivity measured by MARSIS is not absolutely calibrated, we have to 203 compare the reflectivity in the south residual cap to a reference region of known 204 composition.

205 We have chosen a reference region in the SPLD around the position $82^{\circ} \mathrm{S}$ and $150^{\circ} \mathrm{W}$ 206 in a $2^{\circ}$ by $2^{\circ}$ box. It was chosen because of its flatness, so that we can expect that the 207 only parameter that plays a role on the reflectivity is the dielectric constant. We know 208 that the radar waves in the region are reflected by pure water ice, overlain by an 209 optically thin soil layer [Plaut et al. 2007]. In this case, the reflection coefficient of 210 the reference region is estimated as $r_{\text {air } / \mathrm{H}_{2} \mathrm{O}-i c e}=0.279$ with $\varepsilon_{\mathrm{H}_{2} \mathrm{O}-\mathrm{ice}}=3.15$ (see

211 equation 1), which corresponds to a reflectivity $R=\left|r_{\text {air } / \mathrm{H}_{2} \mathrm{O}-i \mathrm{ice}}\right|^{2}=0.078$.

212 In our modeling effort, we consider the south residual cap according to the geological 213 unit defined by Skinner et al. [2006] in the Mars geologic maps. MARSIS 214 measurements cover about $60 \%$ of the $87,000 \mathrm{~km}^{2}$ the south residual cap (to $87^{\circ} \mathrm{S}$ ). 215 We select all MARSIS reflectivity measurements that are either within the south 216 residual cap or in the reference region. We obtain a distribution (see Fig. 4) of the 217 reflectivity for both regions and for each of the three MARSIS frequency bands. The 218 band 1 centered at $1.8 \mathrm{MHz}$ is not used because of the low amount of data. 
219 In order to find the most probable reflectivity values that characterize each region, we

220 fit this distribution by a Gaussian function. Best-fit parameters are summarized in

221 Table 1. Results show that for all frequencies, the reflectivity is much lower in the 222 south residual cap than in the reference region.

223 In order to find the best values for model parameters ( $\varepsilon$, thickness) that reproduce the 224 observations, we use the model described previously. We fix a range for these 225 parameters, which are from 0 to $20 \mathrm{~m}$ for the thickness and from 1.0 to 3.15 for the 226 dielectric constant. The limits for the dielectric constant are the dielectric constant of 227 the upper and lower media (i.e. respectively the atmosphere and the water ice). The 228 procedure consists of a minimization between the model of reflectivity in a stratified 229 medium and the MARSIS measurements for all frequency bands simultaneously.

230 Application of this procedure gives the best value in our model of a mean thickness of $23111 \mathrm{~m}$ and a mean dielectric constant of 2.3. This $\mathrm{CO}_{2}$ dielectric constant is close to the 232 value measured by Pettineli et al. [2003] of 2.12. It confirms that the thin bright slab 233 in the south residual cap is primarily $\mathrm{CO}_{2}$-ice. The formal 1-sigma errors on each 234 parameter, computed from the covariance matrix in the minimization, are $1 \mathrm{~m}$ for the 235 thickness and 0.2 for the dielectric constant.

\subsection{Local Study}

238 In this section, we are not considering the south residual cap as a single unit, but we 239 try to evaluate, locally, (with a resolution of about $14.7 \mathrm{~km}$ ) the properties of the $\mathrm{CO}_{2}$ 240 slab. One can see in Fig. 1c that there is a large variability of reflectivity in the 241 residual cap. 
242 Within our model representation, these variations can be explained as a change in

243 thickness or change in the dielectric constant. In the previous section, we use all the

244 data in the residual cap and so the statistics are robust. This allows us to easily extract

245 the mean behavior. However, in the local study, the statistics for each bin are poor and

246 it is difficult to invert the two parameters at the same time because they play a similar

247 role in reducing the reflectivity of the surface. Alternatively, we can fix one parameter

248 and solve for the other one. Thus for each pixel, we try to describe the reflectivity

249 variation as a change in dielectric constant only, or as a change in $\mathrm{CO}_{2}$-ice thickness

250 only.

\subsubsection{Spatial Variability of the dielectric constant?}

252 First, we fix the thickness at 10 meters and look at the changes in the dielectric 253 constant due to variation of the reflectivity. The resulting dielectric map is shown in 254 Fig. 5a. One can see that the low reflectivity regions (Fig. 1c) correspond to areas 255 where the dielectric constant is close to the value of pure $\mathrm{CO}_{2}$-ice (2.12) [Pettineli et 256 al. 2003]. The central part of the residual cap corresponds to higher values of the 257 dielectric constant, between the $\mathrm{CO}_{2}$-ice (2.12) and $\mathrm{H}_{2} \mathrm{O}$-ice (3.15) reflectivity values, 258 which means in this case a mixture between $\mathrm{H}_{2} \mathrm{O}$-ice and $\mathrm{CO}_{2}$-ice. This mixture could 259 be intimate (at the grain size level) or, because MARSIS has a large footprint, $\mathrm{CO}_{2}$ 260 residual cap and water outcrop reflectivity can be mixed in the returned signal. Using 261 the Maxwell Garnett mixing formula [Sihvola, 1999] and supposing that the effective 262 dielectric constant is only due a mixing between $\mathrm{H}_{2} \mathrm{O}$ - and $\mathrm{CO}_{2}$-ice, we obtain the 263 percentage of $\mathrm{CO}_{2}$-ice compared to $\mathrm{H}_{2} \mathrm{O}$-ice. Fig. 5a shows that the ice content in the 264 central part could be up to $50 \%$ of $\mathrm{H}_{2} \mathrm{O}$, whereas surrounding terrains would contain 265 less than $20 \%$. 


\subsubsection{Spatial Variability of the thickness?}

267 Next, we fix the $\mathrm{CO}_{2}$ dielectric constant at 2.12 and estimate the thickness with our 268 model. This $\mathrm{CO}_{2}$ dielectric constant is close to the previously found value and 269 corresponds to the value measured by Pettineli et al. 2003.

270 Fig. 5b shows the $\mathrm{CO}_{2}$ thickness computed by our reflectivity model. In this 271 hypothesis, we observe on the Fig. $4 \mathrm{~b}$ two types of terrains: terrains with relatively 272 low thickness in the central part (less than 6-7m thick) and higher thickness in the 273 surrounding terrains (about $12 \mathrm{~m}$ thick). As it is difficult to measure a $1 \mathrm{~dB}$ decrease, 274 thicknesses under $4 \mathrm{~m}$ cannot be extracted from our analysis.

\section{Discussion}

\subsection{Errors}

278 In this section, we discuss possible errors in our method.

279 In the first part of the analysis, where we study the general reflectivity of the residual 280 cap, we make an assumption of the constant dielectric of the reference region. For 281 example, if there is a porosity in the shallow subsurface of the ice sheet, the dielectric 282 constant of the reference would decrease and so the reflectivity. For $10 \%$ porosity, $283 \varepsilon_{\mathrm{H}_{2} \mathrm{O}-\mathrm{ice}}$ would be 2.87 and the corresponding reflectivity would be $R=0.066$. A 284 porosity of $10 \%$ in water ice would thus reduce the reflectivity less than $1 \mathrm{~dB}$.

285 Our model assumes no porosity for water ice (i.e. $\varepsilon_{\mathrm{H}_{2} \mathrm{O}-i c e}=3.15$ ) and we think that 286 the assumption does not have a significant effect on our results. Our model is not 287 particularly sensitive to this parameter. For the $10 \%$-porosity case, the output values 288 of the model would be still $11 \mathrm{~m}$ for the thickness and 2.2 for the dielectric constant of 
289

290

291

292

293

294

295

296

297

298

299

300

301

302

303

304

305

306

307

308

309

310

311

312

313 Alternatively, supposing that the residual cap composition is homogeneously pure

the $\mathrm{CO} 2$. This change is inside the uncertainties given by the 1-sigma errors on each parameter.

In the second part, where we study the spatial variability in the south residual cap, we cannot exclude that the effect of roughness at about tens of meters scale could explain the reflectivity variability. However we think that the geologic features in the residual cap (depressions of few meters) are small compared to the MARSIS wavelength and are not responsible for the decrease in reflectivity.

\subsection{Conclusions}

The multi-layered reflection model proposed in this paper allows us to estimate a $\mathrm{CO}_{2}$ slab thickness for a portion of the south residual cap of Mars. The mean $\mathrm{CO}_{2}$ thickness measured by MARSIS seems to be in agreement with the thickness estimated by Thomas et al. 2005.

It is interesting to note that the reflectivity detected by MARSIS is not homogenous across the residual cap. Indeed we observe that the central part of the residual cap has higher reflectivity than surrounding areas.

We have proposed an interpretation of this heterogeneity in terms of dielectric constant and thickness of the $\mathrm{CO}_{2}$-ice slab.

Firstly, supposing that the thickness is constant across the residual cap and solving for dielectric variations, we would conclude that the central part is a mixture of $\mathrm{CO}_{2}$ and $\mathrm{H}_{2} \mathrm{O}$ ices, and the surrounding terrains are mainly pure CO2-ice.

\footnotetext{
Alternatively, supposing that the residual cap composition is homogeneously pure
} 
314 CO2-ice and solving for thickness variations, it appears that the central terrains are

315 thinner that the surrounding terrains. In this case, the volume of $\mathrm{CO}_{2}$-ice contained in

316 the mapped part of the residual cap is about $4.1 \times 10^{11} \mathrm{~m}^{3}$. As MARSIS measurements

317 cover $60 \%$ of the residual cap, we can estimate that the total volume $6.85 \times 10^{11} \mathrm{~m}^{3}$,

318 which corresponds to about 5\% (0.27 mbar) of atmospheric surface pressure (5.6

319 mbar) if we assume that the $\mathrm{CO}_{2}$-ice density is about $1.6 \mathrm{~g} . \mathrm{cm}^{3}$. This estimation is

320 consistent with previous works that predict that the amount of $\mathrm{CO} 2$ in the residual cap

321 is small compared to the mass of the atmosphere [Prettyman et al. 2004; Byrne and

322 Ingersoll, 2003].

323 In both cases, our model shows that the central part of the mapped portion of the

324 residual cap, which shows lower surface reflectivity, contains less $\mathrm{CO}_{2}$-ice than the

325 surrounding parts of the residual cap.

326 A similar analysis could be conducted with the SHAllow subsurface RADar 327 (SHARAD), which operates at $20 \mathrm{MHz}$ (i.e., a wavelength of $15 \mathrm{~m}$ in vacuum). The 328 SHARAD horizontal resolution is $300 \mathrm{~m}$, which would allow description of the 329 surface features at a better resolution. SHARAD may also be sensitive to the seasonal $330 \mathrm{CO}_{2}$ deposits when the thickness is 1-2 $\mathrm{m}$ as described by Nunes and Phillips 2006., 331 although this study is probably more difficult because SHARAD is more sensitive to 332 meter-scale roughness. 


\section{Acknowledgments}

335

336 The authors acknowledge the French space agency (CNES) for support of these 337 studies in Laboratoire de Planétologie de Grenoble. Operations of the Mars Express

338 spacecraft by the European Space Agency (ESA) are gratefully acknowledged. Lynn 339 Carter and an anonymous reviewer provided helpful suggestions for this paper.

340 
341

342

343

344

345

346

347

348

349

350

351

352

353

354

355

356

357

358

359

360

361

362

363

364 365

\section{References}

Bibring, J., and 13 colleagues, 2004. Perennial water ice identified in the south polar cap of Mars , Nature 428, 627-630.

Born M. and Wolf E., 1979. Principles of Optics: Electromagnetic theory of propagation, interference and diffraction of light, Cambridge Univ. Press, Cambridge, UK.

Byrne, S. and Ingersoll, A. P., 2003. A Sublimation Model for Martian South Polar Ice Features, Science 299, 1051-1053.

Douté, S. Schmitt, B., Langevin, Y., Bibring, J.-P., Altieri, F., Bellucci, G., Gondet, B., Poulet, F. \& the MEX OMEGA team, 2007. South Pole of Mars: Nature and composition of the icy terrains from Mars Express OMEGA observations, Planetary And Space Science 55, 113-133.

Duru, F.; Gurnett, D. A.; Averkamp, T. F.; Kirchner, D. L.; Huff, R. L.; Persoon, A. M.; Plaut, J. J. \& Picardi, G., 2006. Magnetically controlled structures in the ionosphere of Mars, J. Geophys. Res. 111, A12204.

Espley, J. R., Farrell, W. M., Brain, D. A., Morgan, D. D., Cantor, B., Plaut, J. J., Acuña, M. H., Picardi, G., 2007. Absorption of MARSIS radar signals: Solar energetic particles and the daytime ionosphere, Geoph. Res. Let. 34, 9101-+. 
367 Flammarion, C., 1892. La planète mars et ses conditions d'habitabilité, v. 1. 368 Gauthier-Villars et Fils, Paris.

369

370 Gurnett, D.A. and 11 colleagues, 2005. Radar soundings of the ionosphere of Mars, 371 Science 310, 1929-1933.

372

373 Kieffer, H.H., 1979. Mars south polar spring and summer temperatures: a residual 374 CO2 frost, J. Geophys. Res. 84, 8263-8288.

375

376

377 378 379 380 381 382 38 384 385 386 387 388

389 Paige, D., Herkenhoff, K., Murray, B., 1990. Mariner 9 observations of the south 390 polar cap of Mars: evidence for residual CO2 frost. J. Geophys. Res. 95, 1319-1335. 
392 Pettinelli, E.; Vannaroni, G.; Cereti, A.; Paolucci, F.; Della Monica, G.; Storini, M. \& 393 Bella, F., 2003. Frequency and time domain permittivity measurements on solid $\mathrm{CO}_{2}$ 394 and solid $\mathrm{CO}_{2}$-soil mixtures as Martian soil simulants, J. Geophys. Res. 108, 10-+ 395

396 Picardi, G. and 12 colleagues, 2004. Performance and surface scattering models for 397 the Mars Advanced Radar for Subsurface and Ionosphere Sounding (MARSIS) 398 Planet. and Space Science 52, 149-156.

399

400 Plaut, J. J.and 23 colleagues, 2007. Subsurface Radar Sounding of the South Polar 401 Layered Deposits of Mars, Science 316, 92-.

402

403 Prettyman, T. H. and 12 colleagues, 2004. Composition and structure of the Martian 404 surface at high southern latitudes from neutron spectroscopy, J. Geophys. Res. 109, 405 5001-+

406

407 Safaeinili, A.; Kofman, W.; Nouvel, J.; Herique, A., Jordan, R. L., 2003. Impact of 408 Mars ionosphere on orbital radar sounder operation and data processing, Planetary 409 and Space Science 51, 505-515.

410

411 Safaeinili, A.; Kofman, W.; Mouginot, J.; Gim, Y.; Herique, A.; Ivanov, A. B.; Plaut, 412 J. J., Picardi, G., 2007. Estimation of the total electron content of the Martian 413 ionosphere using radar sounder surface echoes, Geophysical Reseach Letters 34, 414 23204-+. 
416 Skinner, J.A., Jr., Hare, T.M., Tanaka, K.L., 2006, Digital Renovation of the Atlas of

417 Mars 1:15,000,000-Scale Global Geologic Series Maps, LPSC XXXVII, abstract $418 \quad \# 2331$

419

420 Sihvola A. , 1999. Electromagnetic mixing formulas and applications, IEE 421 Electromagnetic waves series 47, The institution of Electrical Engineers.

422

423

424

425

426 Watters, T. R. and 12 colleagues, 2007. Radar Sounding of the Medusae Fossae

427 Formation Mars: Equatorial Ice or Dry, Low-Density Deposits? 428 Science, 318, 1025.

429

430 Zuber, M. T.; Phillips, R. J.; Andrews-Hanna, J. C.; Asmar, S. W.; Konopliv, A. S.;

431 Lemoine, F. G.; Plaut, J. J.; Smith, D. E. \& Smrekar, S. E., 2007. Density of Mars

432 South Polar Layered Deposits, Science 317, 1718-. 


\section{Tables}

434

\begin{tabular}{l|ccc} 
Center of the Gaussian & $\mathbf{3} \mathbf{~ M H z}$ & $\mathbf{4} \mathbf{~ M H z}$ & $\mathbf{5 ~ M H z}$ \\
\hline South residual cap & -13.1 & -14.1 & -15.5 \\
Surface reference $\left[\mathbf{H}_{\mathbf{2}} \mathbf{O}\right.$-ice] & -7.5 & -8.5 & -8.3 \\
& & & \\
Reflectivity decrease & 5.6 & 5.5 & 7.2
\end{tabular}

435

\section{Table Captions}

437

438 Table 1: The table summarizes the result of the Gaussian fit made on the distribution

439 presented in Fig. 4. The two first lines show the reflectivity (in dB) of the central

440 position of the Gaussian (for the residual cap and for the reference region,

441 respectively). The last line corresponds to the difference (in $\mathrm{dB}$ ) between reference

442 and residual cap. 


\section{Figure Captions}

445

446 Fig. 1: (a) Surface reflectivity map from MARSIS using the radar frequency centered 447 at $4 \mathrm{MHz}$. The projection is polar stereographic. The reflectivity is represented in 448 decibel scale. (b) Simulated reflectivity map using MOLA topography. The 449 450 451 452 453

Fig. 3: Reflectivity $R$ of the layered surface (see Fig. 2) as a function of the thickness $h$ for different values of the dielectric constant $\varepsilon_{\mathrm{CO}_{2}}$. The dielectric constants of the upper and lower medium are 1 and 3.15 , respectively.

Fig. 4: The distribution of surface reflectivity in the reference region (dark grey) and the south residual cap (light grey). The black dashed lines are the Gaussian fit made on the distribution. The results of the fit are summarized in Table 1.

Fig. 5: Maps of the south residual cap region. (a) Map of the dielectric constant found by our reflectivity model with the thickness fixed at 10 meters. Using the Maxwell Garnett mixing formula [Sihvola, 1999] and assuming that the effective dielectric 
468 constant is only due to mixing between $\mathrm{H}_{2} \mathrm{O}$ - and $\mathrm{CO}_{2}$-ice, we give the percentage of

$469 \mathrm{CO}_{2}$-ice and $\mathrm{H} 2 \mathrm{O}$-ice. (b) Map of the $\mathrm{CO}_{2}$ thickness found by our model with 470 dielectric constant fixed at 2.12.

471

472 
Surface reflectivity map at $4 \mathrm{MHz}$ (a)

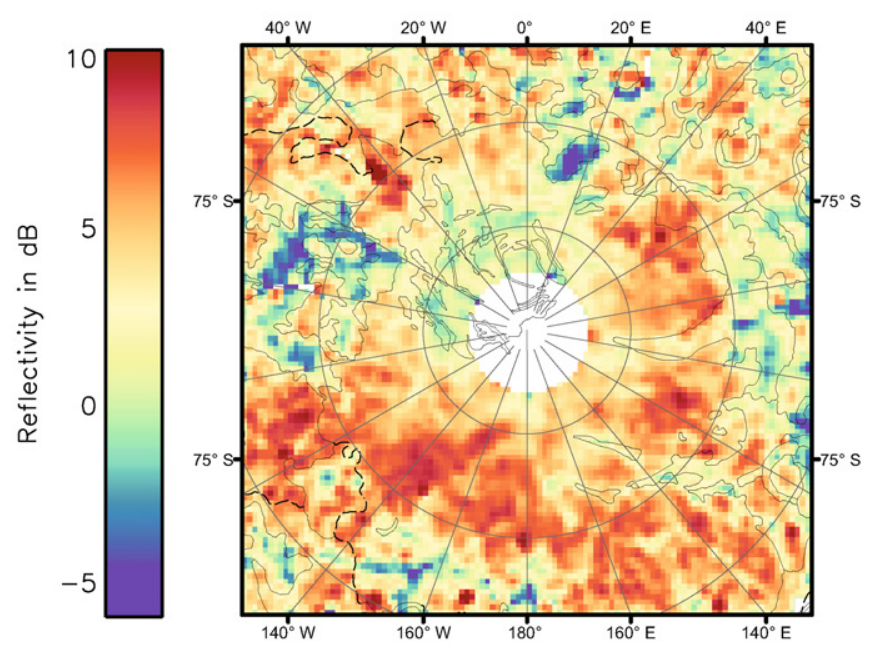

Surface reflectivity normalized by

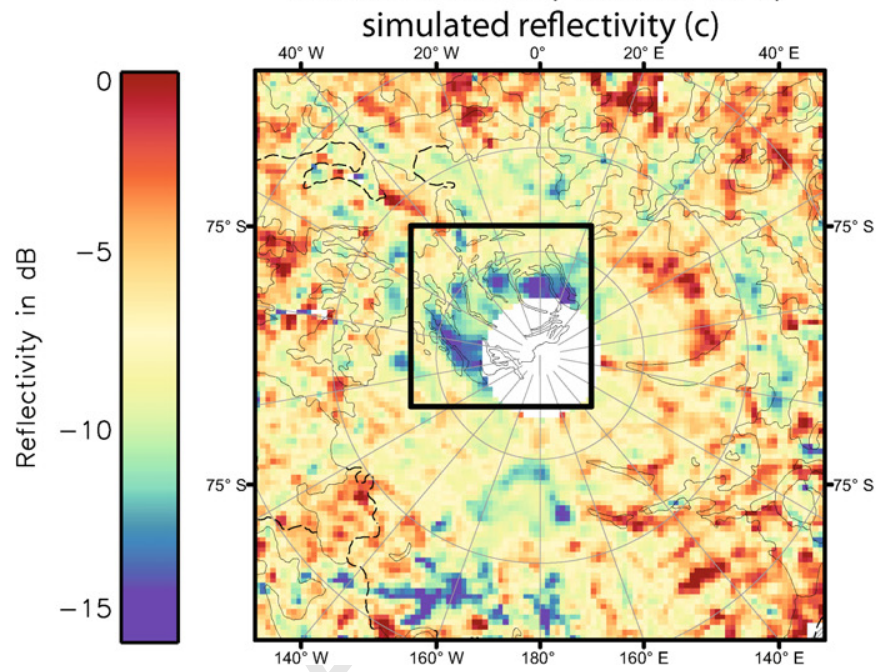

Simulated surface reflectivity (b)
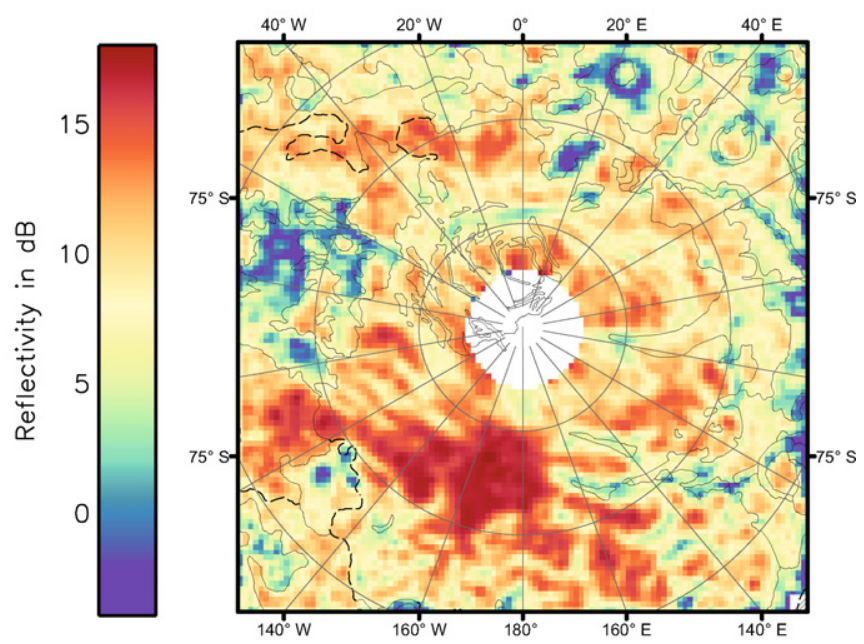

MOC mosaic map (d)

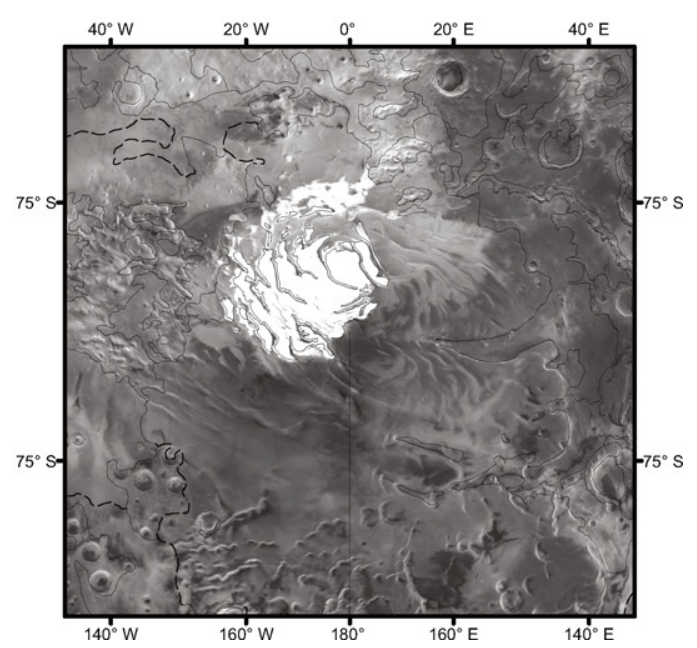


$\varepsilon_{1 \mathrm{r}}=1 \quad$ Atmosphere

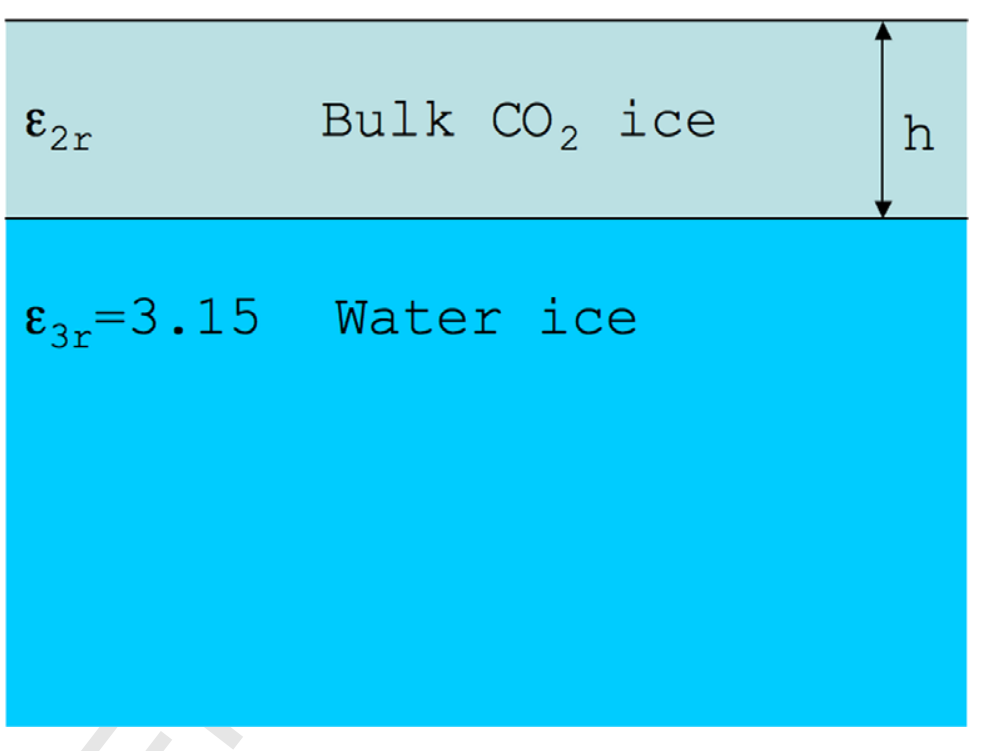




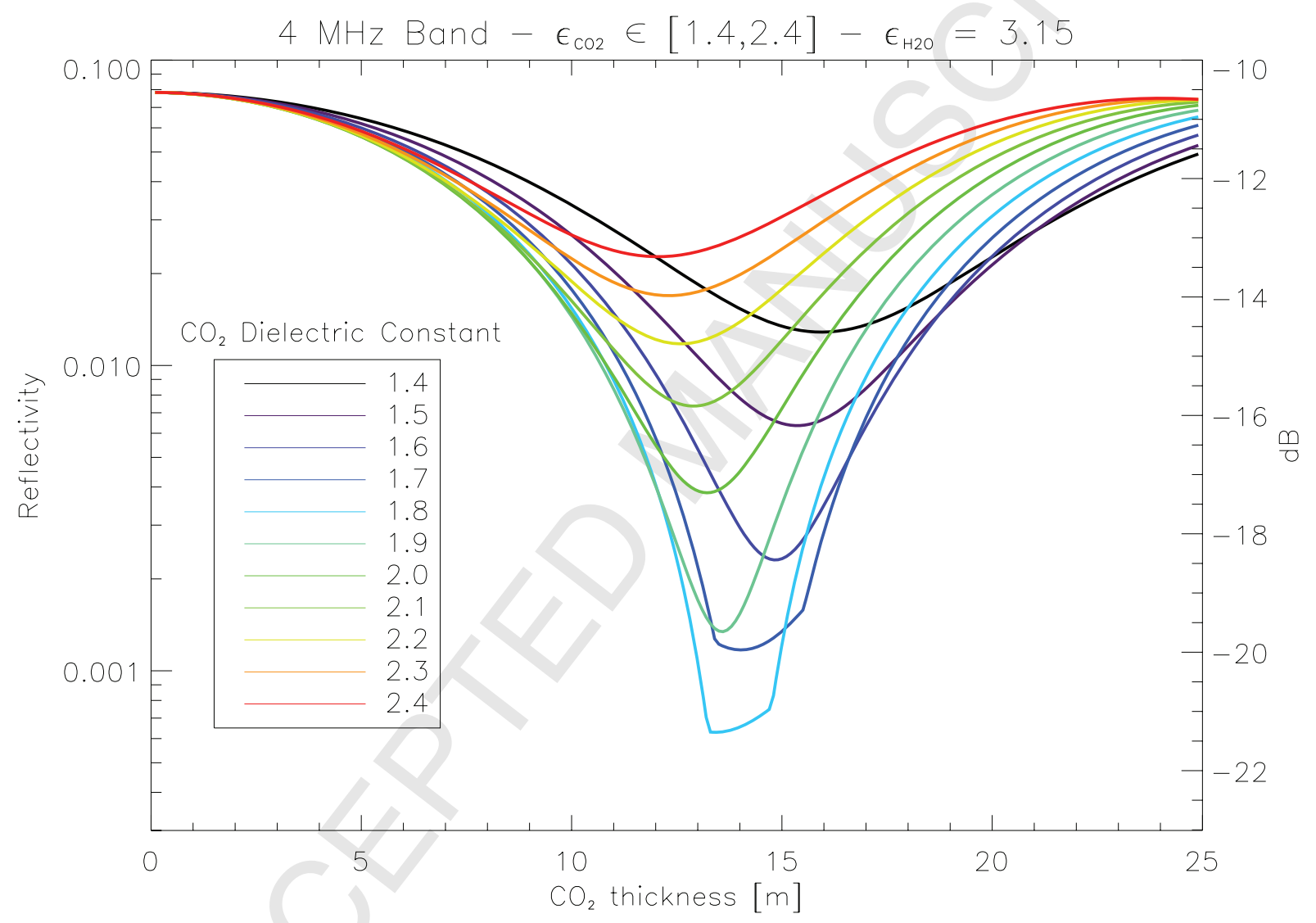




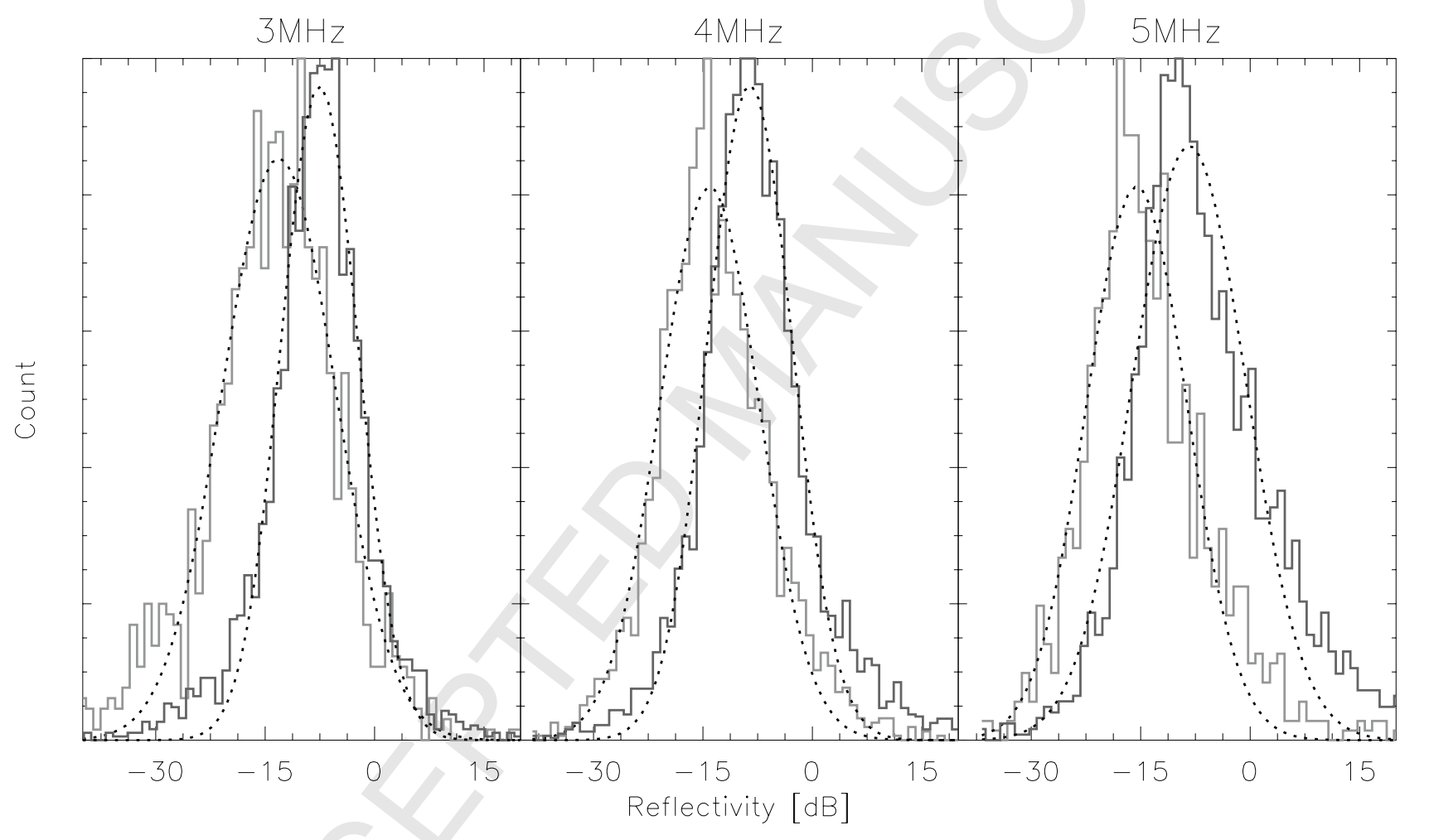



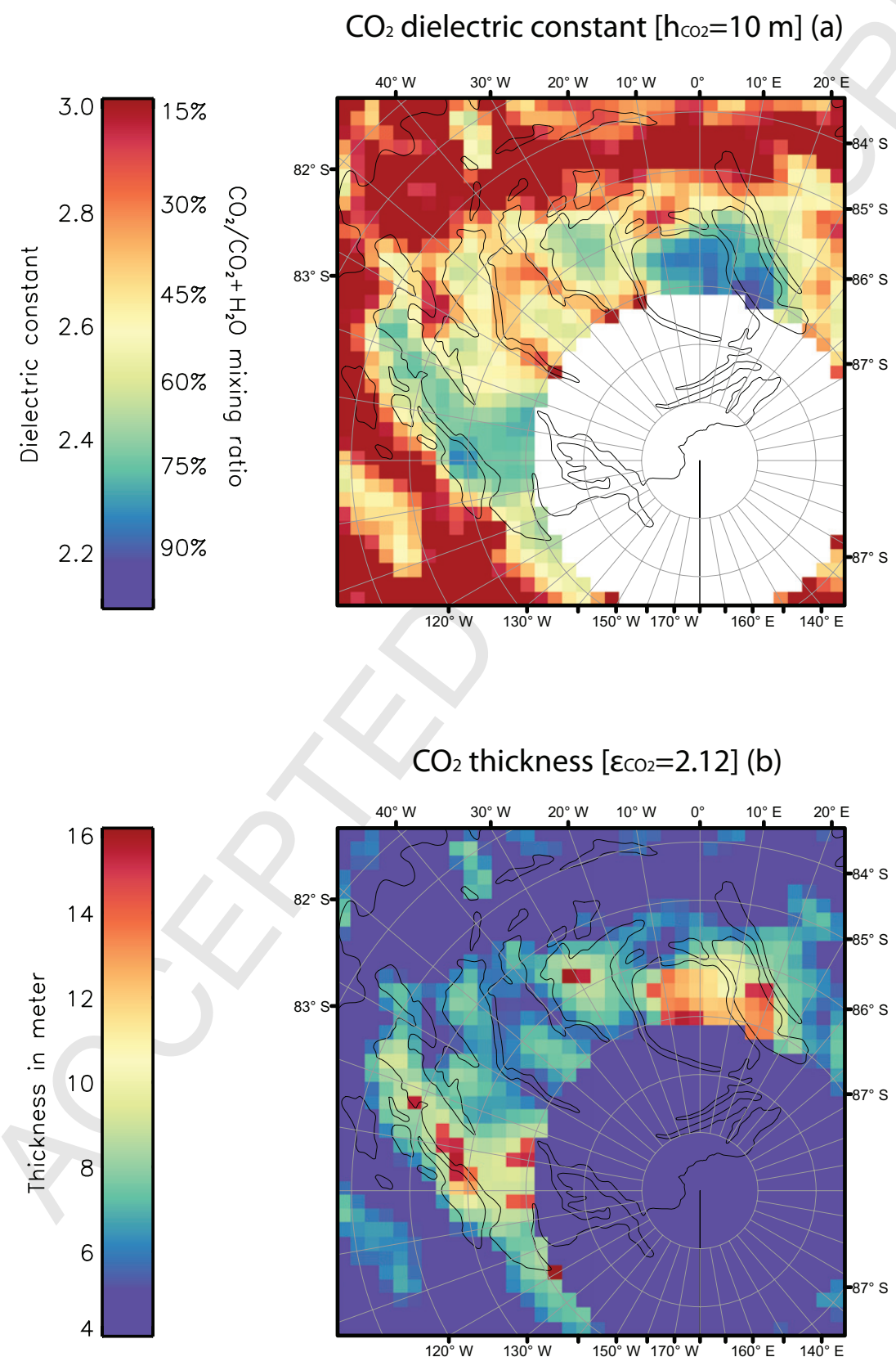

Figure 5 - Mouginot (2008) 